Advancements in Instrumentation

\title{
Air-pressure-driven Separable Microdevice to Control the Anisotropic Curvature of Cell Culture Surface
}

\author{
Tadahiro Yamashita, ${ }^{\dagger}$ Takuya Nishina, Ichiro MATSushita, and Ryo Sudo \\ Department of System Design Engineering, Keio University, 3-14-1 Hiyoshi, Kohoku, Yokohama, \\ Kanagawa 223-8522, Japan
}

\begin{abstract}
We report on a novel microdevice to tune the curvature of a cell-adhering surface by controlling the air-pressure and micro-slit. Human aortic smooth muscle cells were cultured on demi-cylindrical concaves formed on a microdevice. Their shape-adapting behavior could be tracked when the groove direction was changed to the orthogonal direction. This microdevice demonstrated live observation of cells responding to dynamic changes of the anisotropic curvature of the adhering surface and could serve as a new platform to pursue mechanobiology on curved surfaces.
\end{abstract}

Keywords Mechanobiology, curvature, cell adhesion, smooth muscle cell, orientation

(Received January 14, 2020; Accepted March 6, 2020; Advance Publication Released Online by J-STAGE March 20, 2020)

\section{Introduction}

The mechanism of how cells sense the surrounding mechanical environment is one of the core interests in the field so-called mechanobiology. ${ }^{1-3} \quad$ While molecular mechanisms of cellular mechanosensing have been revealed by observing cells confined in various $2 \mathrm{D}$ geometry, there have been an increasing number of studies investigating how cells interact with an artificial 3D environment ${ }^{4-6}$ mimicking the structural complexity in vivo, in pursuit of new medical therapeutics in the future..$^{7-9}$ Among these studies, the effect of the curvature of a cell-adhering surface i.e. concave and convex structures, has been neglected in the field for a long time. However, emerging studies ${ }^{10-13}$ have reported that such curvature has a strong influence on the cellular behaviors including migration, ${ }^{14-16}$ microtissue morphology, ${ }^{17-19}$ and even differentiation, ${ }^{20-22}$ even though the scale of these curved structures is orders of magnitude larger than that of single cells. These studies have clearly shown that the curvature of a cell-adhering plane is a significant input for cells governing their behaviors in a single-cell or continuoustissue scales. However, little is known about the mechanism of how cells sense such a structural cue much larger than single cells.

To unveil the molecular mechanism, live imaging of cells exposed to some dynamic change of the curvature of the adhering plane is desired. However, several technical complexities have prevented researchers from investigating cellular behaviors on micro-curvatures. First, the preparation of a smoothly-curved surface with a sub-millimeter curvature radius is still not feasible, while former studies have utilized a sophisticated fabrication principle, such as the surface tension of a viscous fluid, ${ }^{14}$ mass-transport-limited chemical etching, ${ }^{15}$ stereolithography ${ }^{21,23}$ or an elastic substrate bent by air pressure ${ }^{24-26}$ to prepare micro-curvatures. Especially, a flexible modulation of the micro-curvature pattern, which would be a strong

$\dagger$ To whom correspondence should be addressed.

E-mail: yamashita@sd.keio.ac.jp approach to reveal the adaptation mechanism of cells, remains a challenging issue. Second, a practical limitation in observation, where the objective lens is usually limited to those with a long working distance with typically low magnification due to the thickness of the substrates, often limits live cell imaging. There have been promising microdevices capable of a smooth modulation of the curvature of a cell-adhering surface by air pressure control. $^{25,26}$ However, the lateral geometry of the curving structure is fixed due to the covalently-bound structure of the cell culture surface and the pressure-controlling structure. To realize a flexible control of the curved pattern and live imaging during cell culture, a microdevice design accompanying a non-covalent assembly of the cell-culture surface and the air pressure-controlling system with an optimized thin design for microscopy needs to be developed. Establishing such a new cell-culture system would be valuable to pursue the mechanobiology on curved surfaces.

To meet the increasing demand for a cell culture platform with curvature, we hereby propose a new separable microdevice for live cell monitoring, where the curvature of the cell-adhering plane can be temporarily modulated. The microdevice consists of three components: a cell culture chamber with a thin elastic silicone bottom, a micro-slit with penetrating holes and a thin vacuum chamber. The assembled microdevice modulates the curvature of the cell culture surface by air-pressure control. The separable concept allowing us to reversibly separate and re-assemble the cell culture chamber and an air-pressure controlling system realizes flexible changes of the curvature pattern during the cell culture. As a proof-of-concept study, we demonstrate a time-lapse observation of human aortic smooth muscle cells (SMCs) adhering onto a demi-cylindrical concave surface. Their responses are tracked when the cylindrical direction is turned orthogonally by rotating the cell-culture chamber on the microdevice. This is, to our best knowledge, the first demonstration to observe cells responding to a dynamically changing anisotropic curvature at around the millimeter scale and the microdevice would be a powerful tool to pursue the mechanism of cellular curvature sensing. 


\section{Experimental}

\section{Microdevice design}

A new microdevice was designed for the temporal control of the curvature of a cell-adhering surface (Fig. 1A). It consists of three components: a cell culture chamber (Fig. 1B), a micro-slit (Fig. 1C) and a vacuum chamber (Fig. 1D). The supplementary material should be referred to for the detailed design and the fabrication process. Cells are cultured on an elastic silicone sheet forming the bottom part of the cell culture chamber. By applying a negative air pressure from the vacuum chamber to the sheet through the micro-slit, the cell culture surface can be bent. The vacuum chamber with a glass window is designed to be thin enough for live microscopic observations of cultured cells. These components were assembled and set in a stage incubation system (GM-8000 and WSKMOR-GI, TOKAI HIT, Fujinomiya, Japan) installed on a microscope (Fig. 1E). The hole made in the sidewall of the vacuum chamber was connected to a vacuum pump (DAP-6D, ULVAC, Chigasaki, Japan) via a dumping chamber (FB-800-5000, ASONE, Osaka, Japan) and a pressure controller (VC900, KNF, Freiburg, Germany) (Fig. 1F).

\section{Calibration of curvature generated by an air-pressure control}

The curvature of the silicone sheet generated by air pressure was calibrated using a phase-contrast microscope (Eclipse Ti, Nikon, Tokyo, Japan). The heights of the bottom and the top parts of the deformed silicone sheet were measured. Approximating the cross-sectional shape of the deformed silicone sheet to an arc, the curvature was calculated. The differential pressure $\Delta P$ was tuned from 0 to $600 \mathrm{hPa}$ using the pressure controller. Cylindrical and spherical concave surfaces were formed using micro-slits containing rectangular (300 and $500 \mu \mathrm{m}$ in width) and circular (300 and $500 \mu \mathrm{m}$ in diameter) holes, respectively. The cross-sectional shape of the deformed silicone sheet was also observed via confocal microscopy. Supplementary material should be referred to for the details of the curvature measurement.

\section{Cell culture using a microdevice}

Suspended SMCs were seeded on the bottom of a cell culture chamber covalently coated with fibronectin at $7 \times 10^{4}$ and $3 \times 10^{4}$ cells $/ \mathrm{cm}^{2}$ under high- and low-density conditions, respectively, followed by incubation for $12 \mathrm{~h}$ to allow cells to form stable adhesion. The supplementary material should be referred to for the experimental details. SMCs were treated with Hoechst 33342 (1:10000, H3570, Thermo Fisher, Waltham, MA, USA) in a serum-free cell-culture medium for $5 \mathrm{~min}$ and the chamber was refilled with the cell culture medium with growth factors. The SMC-containing cell culture chamber was mounted on a micro-slit with three rectangular holes (width, $500 \mu \mathrm{m}$; length, $6000 \mu \mathrm{m}$ ) and the vacuum chamber installed inside the stage incubation system. The differential pressure was gradually increased to $100 \mathrm{hPa}$, taking $100 \mathrm{~min}$ to bend the cell-adhering surface to form a demi-cylindrical concave without applying any direct strain on the SMCs. The SMCs were cultured for $24 \mathrm{~h}$. Following pressure release, taking $100 \mathrm{~min}$, the medium was exchanged. The cell culture chamber was then carefully detached from the micro-slit, rotated $90^{\circ}$ horizontally and placed on it again so that the three rectangular holes of the micro-slit under the cell culture chamber would be positioned orthogonally to the original direction. Observing the positions of tiny bubbles and particles in the wall of the cell culture chamber under microscope, the alignment of the assembled components as well as the area of interest where a demi-

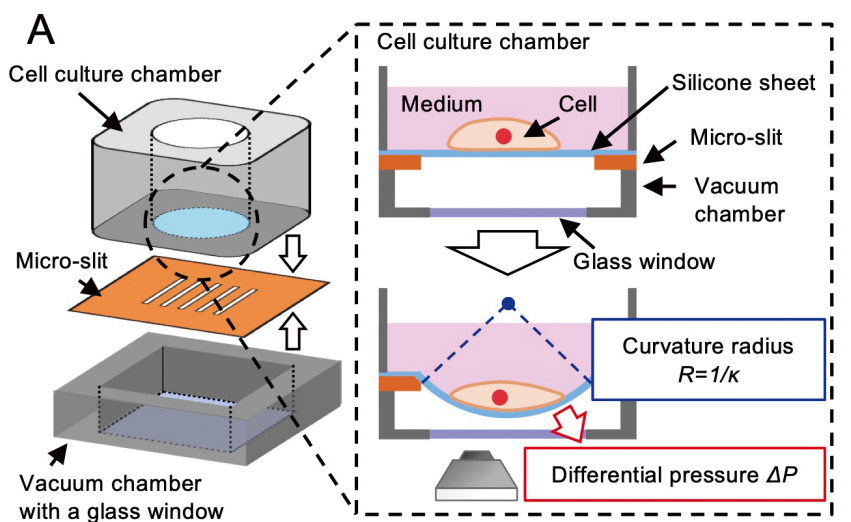

with a glass window

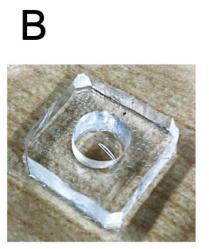

C

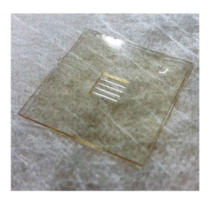

D

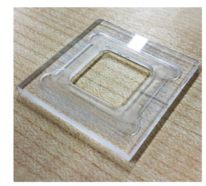

$\mathrm{E}$

F

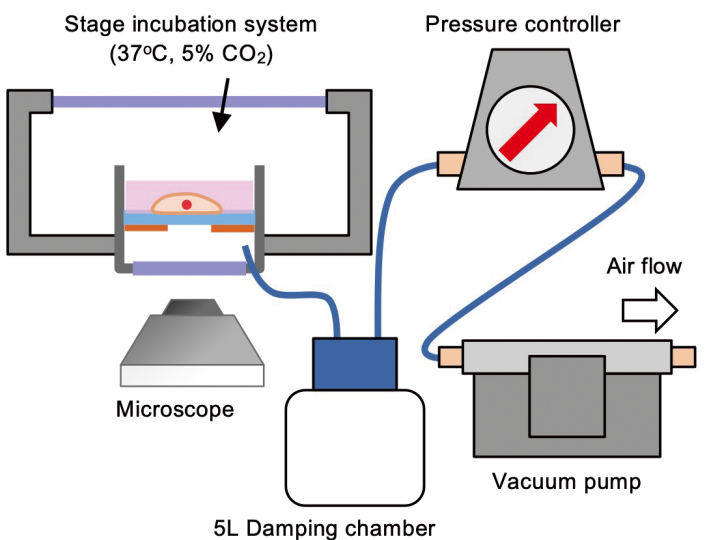

Fig. 1 Design of microdevice. (A) The microdevice is composed of a cell culture chamber with a thin bottom made of silicone sheet, a micro-slit made of SU-8 and a vacuum chamber with a glass bottom Cells are cultured on the thin silicone sheet and the curvature of the adhering plane can be temporarily controlled by tuning the air pressure inside the vacuum chamber. The cellular response can be observed by microscope in real time. (B) Cell culture chamber with a thin silicone bottom. (C) SU-8 micro-slit containing three straight penetrating holes in the centre (width, $500 \mu \mathrm{m}$; length, $6 \mathrm{~mm}$ ). (D) Vacuum chamber with glass window. (E) Assembled microdevice containing cells and cell culture medium installed on the microscopic stage. (F) Schematic of the air-pressure control system. The microdevice installed in the stage incubation system was connected to a vacuum pump via a pressure controller that allows flexible modulation of the differential pressure applying to the cell culture surface.

cylindrical-concave situation was formed before rotating the cell culture chamber was carefully checked. The differential pressure was again increased to $100 \mathrm{hPa}$, as described above, followed by incubation for an additional $24 \mathrm{~h}$. Nuclei of the SMCs were observed using the fluorescence microscope with either a $10 \times($ N.A. 0.30$)$ or a $20 \times($ N.A. 0.45$)$ lens every $6 \mathrm{~h}$. The nuclear orientation was analyzed using a custom script written in MATLAB (2016b, Mathworks, Natick, MA, USA). In brief, the nuclear shape was extracted by thresholding and binarizing the fluorescence image. Each nucleus was then fitted to an ellipse and the angle between the long axis of the ellipse and the axis of the demi-cylindrical structure was evaluated. 
A

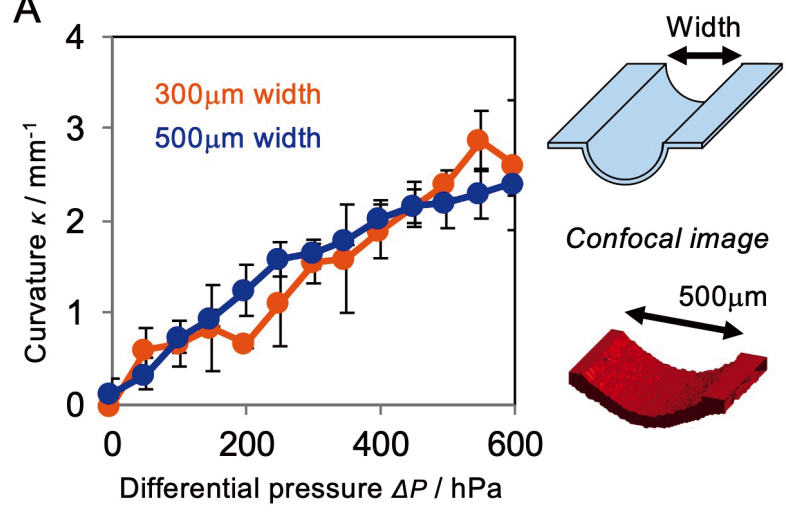

B

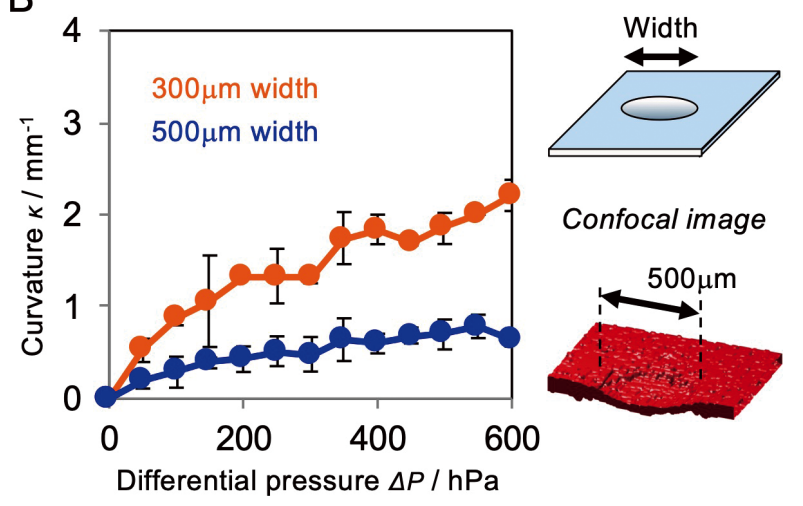

Fig. 2 Calibration of the curvature tuned by an air-pressure control. The curvature of a demi-cylindrical surface (A) and a demi-spherical surface (B) generated using the micro-slits with rectangular holes and the one with circular holes, respectively, was calibrated as a function of the applied differential pressure. Representative 3D structures observed in the experiment were reconstructed from stacks of confocal images. The error bars represent the standard deviations of three different silicone sheets.

\section{Results and Discussion}

The microdevice components were assembled by mounting the cell culture chamber onto the vacuum chamber via micro-slits with rectangular penetrating holes. By sucking the air from the vacuum chamber, the silicone sheet forming the bottom of the cell culture chamber was bent to form demi-cylindrical concaves. The curvature of the silicone sheet was first characterized by varying the applied pressure. The curvature linearly increased as the differential pressure was elevated from 0 to $400 \mathrm{hPa}$, and then gradually reached $2.5 \mathrm{~mm}^{-1}$ (Fig. 2A). The curvature could be smoothly tuned over the range of $0-2 \mathrm{~mm}^{-1}$, corresponding to from a flat state to a demi-cylindrical surface of $500 \mu \mathrm{m}$ radius. Cross-sectional views obtained by confocal microscopy confirmed that the silicone sheet formed a smooth arc without any wrinkles or buckles (Fig. S6 in SI). Using the micro-slit containing circular holes, concave pits could also be formed on the bottom of the cell culture chamber (Fig. 2B). While the curvature-differential pressure relations obtained using 300 and $500 \mu \mathrm{m}$ rectangular micro-slits were similar, a considerable difference was observed between those obtained using 300 and $500 \mu \mathrm{m}$ circular micro-slits. Because rectangular and circular micro-slits induce uniaxial and biaxial stretch, respectively, we assumed that the difference in the stretching modes caused such a different dependence on the micro-slit size. After forming and releasing the demi-cylindrical concave of $1 \mathrm{~mm}^{-1}$ curvature, no crack, which potentially influences the cellular alignment, was found on the silicone-sheet surface via scanning electron microscopy (Fig. S7 in SI). Throughout these experiments, we confirmed that this separable micro-slit device could form a smoothly curved surface on the cell culture chamber.

The microdevice was next applied for a cell culture. Although the SMCs initially oriented to all directions randomly on the flat silicone sheet, they preferentially aligned along the cylindrical axis $22.5 \mathrm{~h}$ after forming the demi-cylindrical concave (Fig. 3A). Next, the cell-culture chamber was rotated orthogonally to the original direction on the micro-slit to observe how the SMCs react to a sudden directional change of the anisotropic curvature of the adhering surface. SMCs were exposed to a demicylindrical concave perpendicular to the original one and they gradually aligned along the new structure within $24 \mathrm{~h}$, reproducing the same distribution profile observed prior to the chamber rotation (Fig. 3B). Representative nuclear images at each time point under high- and low-density conditions are summarised in Figs. S8 and S9 in SI, respectively.

The SMCs on the demi-cylindrical concave in the high-density condition always adjusted their orientation parallel to the cylindrical axis (Fig. 3C), which accords well to the common knowledge in the field that cells elongate along given structural patterns when there is an anisotropic chemical or physical pattern on the adhering surface. ${ }^{27}$ This is also supported by theoretical and numerical studies explaining the optimal angle based on minimization of cytoskeltal strain $^{28}$ and the planer shear stress. ${ }^{29}$ However, the trend is less clear under the lowdensity condition (Fig. 3D), where SMCs showed a more random orientation throughout the cell culture period, although a considerable portion of SMCs oriented along the demicylindrical axis after turning the cylindrical axis. Comparing the orientation profiles under both conditions, it is likely that intercellular connection helps the cells to find a proper direction to align on the curved structures on the millimeter scale.

In principle, our platform cannot separate the effects of the substrate curvature and the stretch on the cellular orientation. However, the stretching effect could be negligible in our case, because the circumferential strain of the silicone sheet when forming the curvature was around $1 \%$ and it was slowly operated, taking $100 \mathrm{~min}$, while the typical stretching mode, known to influence SMC orientation, was a 5-10\% cyclic stretch of frequency above $0.5 \mathrm{~Hz} \cdot{ }^{30,31}$ We thus think that the re-orientation behavior of SMCs was caused by a structural change of the adhering surface. A former study conducted by Ebara and colleagues applied a perpendicular transition of a striped surface topographic pattern on a sub-micron scale to sparsely cultured fibroblasts utilising a shape memory polymer. ${ }^{32}$ They also reported that the cells suddenly exposed to the new topological cue adapted to the new pattern within a scale of one day. Our observation accords well to their report, suggesting that a common or quite similar mechanism underlies the cellular curvature sensing on a curved surface and topological sensing on a $2 \mathrm{D}$ surface. The effect of the cellular density and the difference in the cell types on the curvature sensing need to be investigated to explore the mechanism in the future.

Our microdevice demonstrated that SMCs could sense a subtle anisotropic curvature of $1 \mathrm{~mm}^{-1}$, corresponding to $1 \mathrm{~mm}$ of the curvature radius, and adapt to directional changes of the cylindrical cell-adhering surface. There is so far no general threshold of the lower limit of cellular curvature sensing in the field, ${ }^{11}$ and it is notable that cells sense curvature whose scale is orders of magnitude larger than that of a single cell. The mechanism of how connected cells sense the surrounding 
A
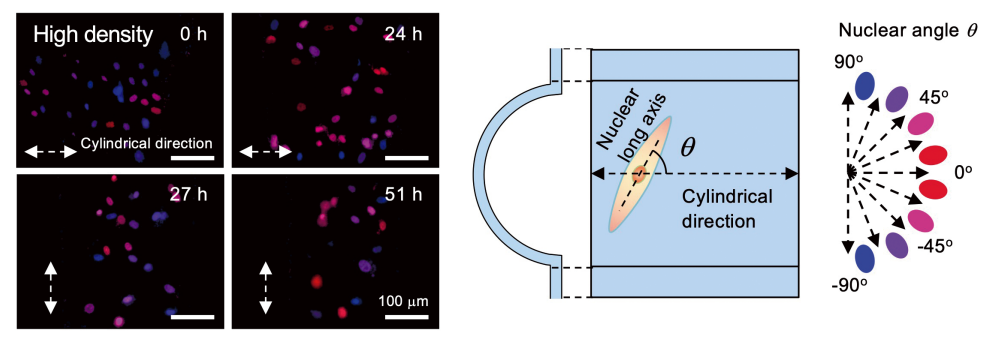

B
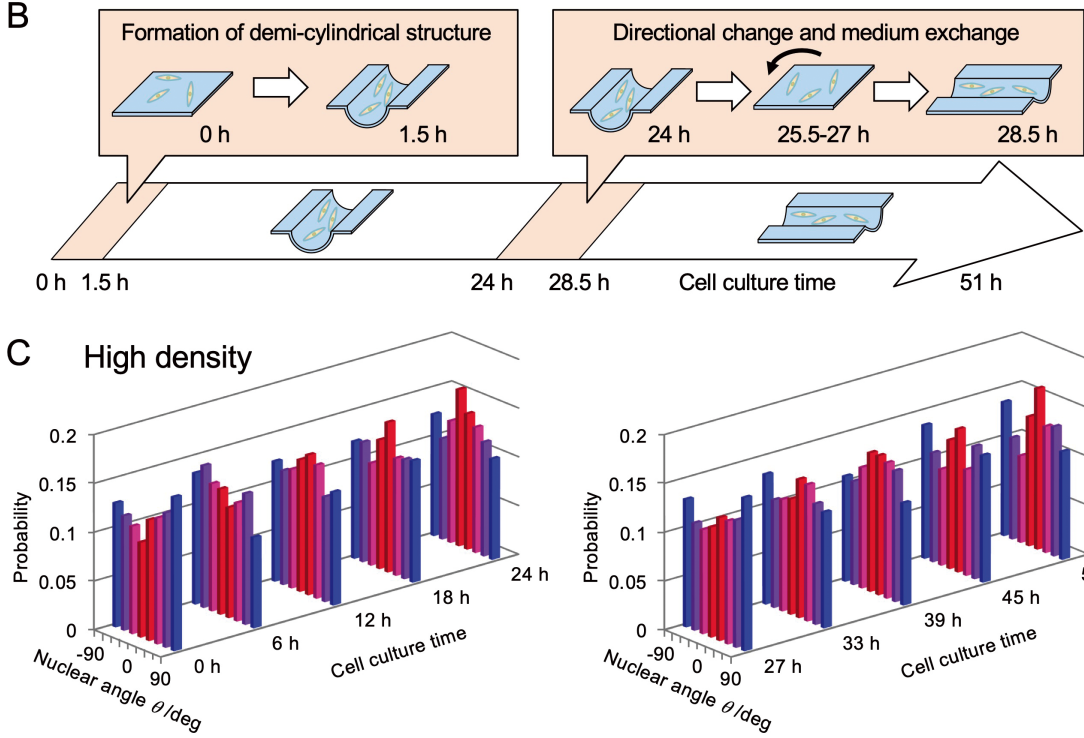

D
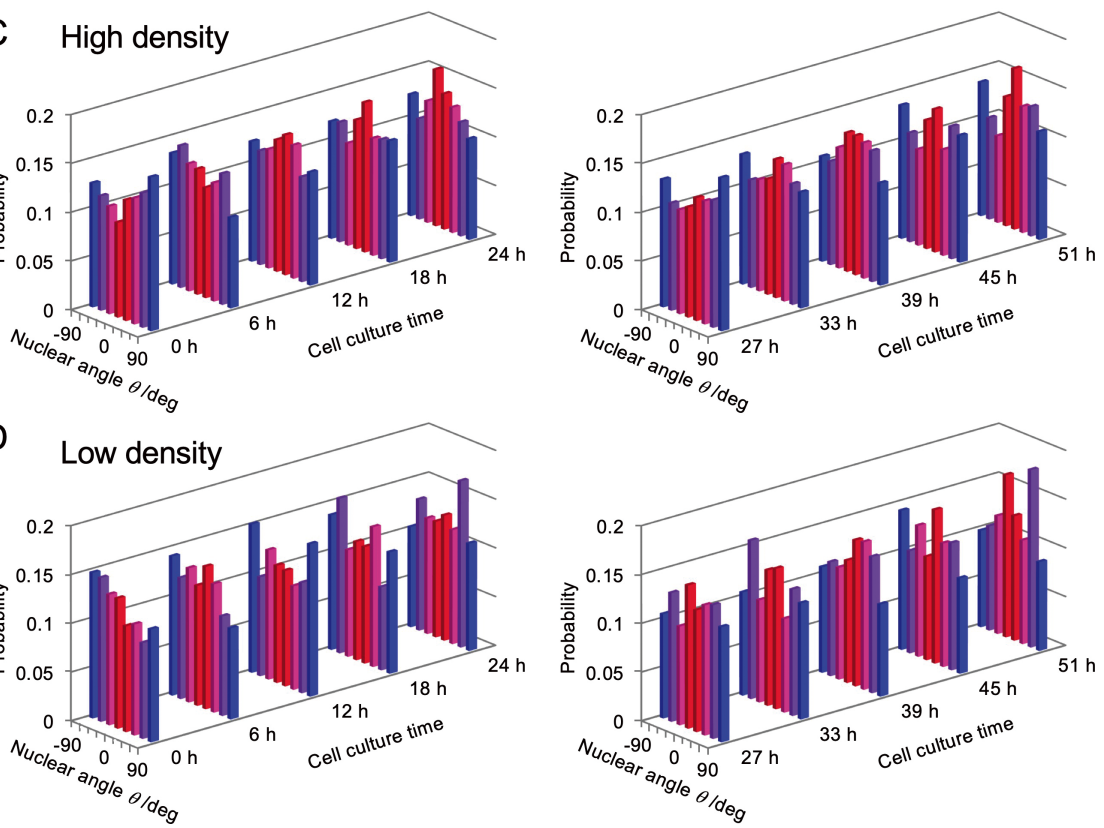

Fig. 3 Curvature-transition assay applied for human aortic SMCs. The transition of the nuclear orientation was analyzed. (A) Nuclei of Hoechst-treated SMCs were imaged and the angle between the long axis of each nucleus and the cylindrical direction was evaluated as nuclear angle. Each nucleus is colored according to the nuclear angle. (B) The cell culture surface was bent to form a demi-cylindrical surface of $1 \mathrm{~mm}^{-1}$ of curvature in the initial $1.5 \mathrm{~h}$. The axial direction of the demi-cylindrical concave was turned orthogonally to the initial direction $24 \mathrm{~h}$ from starting the experiment. Nuclear alignment of SMCs were tracked for $51 \mathrm{~h}$. (C, D) Transition of nuclear orientation of SMCs were analyzed. More than 1000 and 250 nuclei in high-density (C) and low-density (D) conditions, respectively, were analyzed at each time point. The experiments were duplicated to ensure the reproducibility.

environment, ${ }^{33}$ as well as their curvature sensing ability, has still not been explored. Our platform would serve the needs to give steric perturbations to cells, together with either static ${ }^{34}$ or dynamic $^{35}$ cell-confining methods on 2D flat surfaces. Conducting the curvature-transition assay with biological inhibitors for cell-adhesion-related proteins, the mechanism of how cells sense and respond to the surrounding dynamic topographic change could be revealed.

From an engineering perspective, our separable microdevice is unique as a cell-culture platform to realize dynamic topographic change of the cell culture environment. Various microfluidic vascular models have been widely developed in the field of Micro Total Analysis Systems. ${ }^{36,37}$ While biomechanical stimuli including fluid shear stress and cyclic stretch have been implemented in these microvascular models, the effect of the substrate curvature that potentially affects cellular properties, ${ }^{10-13}$ such as motility and phenotype has not been highlighted so far. On the other hand, a topographic change of the cell-adhering surface on a sub-micron scale was already realized in the biomaterial field using a polymeric shape-memory effect triggered by some temperature change. ${ }^{32}$ While such a chemical change can be triggered only once, which requires temperature change that inevitably affects on cellular metabolism, our system enables dynamic control of the anisotropic curvature without changing the temperature and it, in principle, does not have any limitation in repeating this topographic change. There have been several cell-culture platforms that control the air pressure to apply some cyclic stretch to cells, ${ }^{25}$ which can be 
potentially applied to tune the curvature of the cell-adhering plane. However, the geometry of the curved structure is fixed due to irreversible bonding of the elastic membrane and the vacuum structure. On the other hand, our separable microdevice is capable of flexibly changing the design of the curved part by replacing the micro-slit. The current microdevice is limited to create concave structures because of the working principle based on negative air pressure. However, the convex curvature could be potentially realized by applying reversible bonding techniques for microfluidics. ${ }^{38,39}$ Although there is a tradeoff relation between the range of the tunable curvature, the tunable area and the thickness of the vacuum chamber limiting the usage of high-NA lenses, further high-resolution imaging of the cells on a curved surface could be potentially realized by compromising the flexibility in the curvature-tuning. Enriching the toolbox currently limited to investigate cellular behaviors on a curved surface, our microdevice would contribute to the mechanobiology field.

\section{Acknowledgements}

This study was supported by KAKENHI (Grant Numbers $18 \mathrm{H} 05963$ and 19K20679) and bilateral programs from Japan Society for the Promotion of Science, Keio Gijuku Academic Development Funds and Keio Leading-edge Laboratory grant. We would like to thank Prof. Kenjiro Takemura (Keio University) and Dr. Chikahiro Imashiro (now Tokyo Women's Medical University) for their kind support with machining.

\section{Supporting Information}

The details on microdevice fabrication and cell culturing are described in a supporting material. This material is available free of charge on the Web at http://www.jsac.or.jp/analsci/.

\section{References}

1. K. A. Jansen, D. M. Donato, H. E. Balcioglu, T. Schmidt, E. H. J. Danen, and G. H. Koenderink, Biochim. Biophys. Acta, Mol. Cell Res., 2015, 1853, 3043.

2. N. I. Petridou, Z. Spiró, and C. P. Heisenberg, Nat. Cell Biol., 2017, 19, 581.

3. T. J. Kirby and J. Lammerding, Nat. Cell Biol., 2018, 20, 373.

4. N. Huebsch, Acta Biomater, 2019, 94, 97.

5. H. Liu, Y. Wang, K. Cui, Y. Guo, X. Zhang, and J. Qin, $A d v$. Mater., 2019, 31, 1902042.

6. M. Rumpler, A. Woesz, J. W. C. Dunlop, J. T. van Dongen, and P. Fratzl, J. R. Soc. Interface, 2008, 5, 1173.

7. W. R. Thompson, A. Scott, M. T. Loghmani, S. R. Ward, and S. J. Warden, Phys. Ther., 2016, 96, 560.

8. J. L. Ng, M. E. Kersh, S. Kilbreath, and M. Knothe Tate, Front. Physiol., 2017, 8, 303.

9. M. C. Lampi and C. A. Reinhart-King, Sci. Transl. Med., 2018, 10, eaao0475.

10. J. Foolen, T. Yamashita, and P. Kollmannsberger, J. Phys. $D$, 2016, 49, 053001.

11. D. Baptista, L. Teixeira, C. van Blitterswijk, S. Giselbrecht, and R. Truckenmüller, Trends Biotechnol., 2019, 37, 838.
12. R. K. Assoian, N. D. Bade, C. V. Cameron, and K. J. Stebe, Open Biol., 2019, 9, 190155.

13. S. J. P. Callens, R. J. C. Uyttendaele, L. E. Fratila-Apachitei, and A. A. Zadpoor, Biomaterials, 2020, 232, 119739.

14. N. D. Bade, T. Xu, R. D. Kamien, R. K. Assoian, and K. J. Stebe, Biophys. J., 2018, 114, 1467.

15. L. Pieuchot, J. Marteau, A. Guignandon, T. D. Santos, I. Brigaud, P. F. Chauvy, T. Cloatre, A. Ponche, T. Petithory, P. Rougerie, M. Vassaux, J. L. Milan, N. T. Wakhloo, A. Spangenberg, M. Bigerelle, and K. Anselme, Nat. Commun., 2018, 9, 3995.

16. M. Werner, A. Petersen, N. A. Kurniawan, and C. V. C. Bouten, Adv. Biosyst., 2019, 3, 1900080.

17. K. E. Broaders, A. E. Cerchiari, and Z. J. Gartner, Integr. Biol., 2015, 7, 1611.

18. T. Yamashita, P. Kollmannsberger, K. Mawatari, T. Kitamori, and V. Vogel, Acta Biomater, 2016, 45, 85.

19. S. Ehrig, B. Schamberger, C. M. Bidan, A. West, C. Jacobi, K. Lam, P. Kollmannsberger, A. Petersen, P. Tomancak, K. Kommareddy, F. D. Fischer, P. Fratzl, and J. W. C. Dunlop, Sci. Adv., 2019, 5, eaav9394.

20. T. Ozdemir, L. C. Xu, C. Siedlecki, and J. L. Brown, Integr. Biol., 2013, 5, 1407.

21. M. Werner, S. B. G. Blanquer, S. P. Haimi, G. Korus, J. W. C. Dunlop, G. N. Duda, Dirk. W. Grijpma, and A. Petersen, Adv. Sci., 2016, 4, 1600347.

22. P. Kollmannsberger, C. M. Bidan, J. W. C. Dunlop, P. Fratzl, and V. Vogel, Sci. Adv., 2018, 4, eaao4881.

23. D. Cheng, R. K. Jayne, A. Tamborini, J. Eyckmans, A. E. White, and C. S. Chen, Biofabrication, 2019, 11, 021001.

24. J. Y. Park, D. H. Lee, E. J. Lee, and S. H. Lee, Lab Chip, 2009, 9, 2043.

25. J. Zhou and L. E. Niklason, Integr. Biol., 2012, 4, 1487.

26. N. Venugopal Menon, H. M. Tay, K. T. Pang, R. Dalan, S. C. Wong, X. Wang, K. H. H. Li, and H. W. Hou, APL Bioeng., 2018, 2, 016103.

27. H. Jeon, C. G. Simon, and G. Kim, J. Biomed. Mater. Res., Part B, 2014, 102, 1580.

28. Y. Y. Biton and S. A. Safran, Phys. Biol., 2009, 6, 046010.

29. C. Liu, J. Xu, S. He, W. Zhang, H. Li, B. Huo, and B. Ji, J. Mech. Behav. Biomed. Mater., 2018, 88, 330.

30. P. R. Standley, A. Camaratta, B. P. Nolan, C. T. Purgason, and M. A. Stanley, Am. J. Physiol. Heart Circ. Physiol., 2002, 283, H1907.

31. B. Liu, M. J. Qu, K. R. Qin, H. Li, Z. K. Li, B. R. Shen, and Z. L. Jiang, Biophys. J., 2008, 94, 1497.

32. M. Ebara, K. Uto, N. Idota, J. Hoffman, and T. Aoyagi, Int. J. Nanomed., 2014, 9, 117.

33. B. Ladoux and R. M. Mège, Nat. Rev. Mol. Cell Biol., 2017, 18, 743.

34. N. Tanaka, T. Yamashita, A. Sato, V. Vogel, and Y. Tanaka, PLoS ONE, 2017, 12, e0173647.

35. J. Nakanishi, K. Sugiyama, H. Matsuo, Y. Takahashi, S. Omura, and T. Nakashima, Anal. Sci., 2019, 35, 65.

36. K. Sato, A. Kodama, C. Kase, S. Hirakawa, and M. Ato, Anal. Sci., 2018, 34, 323.

37. K. Sato and K. Sato, Anal. Sci., 2018, 34, 755.

38. L. S. Shiroma, M. H. O. Piazzetta, G. F. Duarte-Junior, W. K. T. Coltro, E. Carrilho, A. L. Gobbi, and R. S. Lima, Sci. Rep., 2016, 6, 1.

39. M. Chu, T. T. Nguyen, E. K. Lee, J. L. Morival, and M. Khine, Lab Chip, 2017, 17, 267. 